\title{
Multiple HIV-1 Genotypes Circulating Among College Students in Nanjing, China
}

\author{
Wei Li, ${ }^{1, *}$ Zhengping Zhu, ${ }^{2, *}$ JinJin Chu, ${ }^{1, *}$ You Ge, ${ }^{1}$ Yuanyuan Xu, ${ }^{2}$ Sushu Wu, ${ }^{2}$ Yan He, \\ Qian Ni, ${ }^{1}$ Yangyang Liu, ${ }^{1}$ Rui Li, ${ }^{1}$ Xiaoshan $\mathrm{Li}^{3}$ and Pingmin $\mathrm{Wei}^{1}$
}

\begin{abstract}
College students are disproportionately affected by HIV-1 in China. However, little is known about the genetic characteristics of HIV-1 among this population. A molecular epidemiological investigation was conducted among the newly diagnosed antiretroviral therapy-naïve HIV-1-infected individuals during 2015-2019 in Nanjing city, China. The pol fragment (HXB2: 2,253-3,311) was obtained by HIV-1 RNA extraction and gene amplification, and subjected to genotyping, recombination analysis, and phylogenetic inference. A total of $945 \mathrm{pol}$ sequences from 226 students and 719 nonstudents were successfully amplified. Multiple genotypes were identified in students, including CRF01_AE (37.66\%), CRF07_BC (32.90\%), CRF55_01B (5.63\%), CRF68_01B (3.46\%), CRF67_01B (3.03\%), subtype B (1.73\%), and CRF58_01B (1.30\%) and unique recombinant forms (URFs) of 01C_like (7.08\%), 0107_like (3.98\%), 01BC_like (2.21\%), and 01B_like (1.33\%). The distribution of genotypes among students was similar to that among nonstudents. The estimated mean evolutionary rate of URFs was $2.89 \times 10^{-3}$ [95\% Bayesian credible interval: 1.89-3.90] nucleotide substitutions/site/year. Approximately 64\% (21/33) of URFs among students were located in three major clusters (0107_like, 01C_like 1, and 01C_like 2 clusters), which had recent time to the most recent common ancestors and low mean genetic distance, and presumably originated from Nanjing (posterior probability $\geq 0.99$, state probability $\geq 0.9$ ). Among 226 students with pol segments, the prevalence of primary and transmitted drug resistance mutations was $15.93 \%$ and $3.98 \%$, respectively. The rapid evolution of multiple HIV-1 genotypes and high prevalence of URFs circulating among students in Nanjing emphasized the necessity of comprehensive surveillance for HIV-1 transmission among this population.
\end{abstract}

Keywords: HIV-1, college students, genotype distribution, phylogenetic analysis

\section{Introduction}

A DOLESCENTS (10-19 YEARS) continue to be disproportionately affected by HIV/AIDS globally. The number of adolescents living with HIV/AIDS has risen by $30 \%$ between 2005 and $2016,{ }^{1}$ with an estimated 1.8 million adolescents being infected with HIV worldwide in $2017 .^{2}$ The number of adolescents dying due to AIDS-related illnesses tripled between 2000 and 2015, and 37,977 adolescents died from AIDS-related causes in 2017. Among all HIV-affected populations, adolescents are the only group for which the mortality figures are not decreasing. ${ }^{3}$ AIDS is now the second leading cause of death among young people worldwide. ${ }^{4}$
In China, young people were not previously considered highly susceptible to HIV-1 infection, ${ }^{5}$ until recently, the number of individuals infected with HIV-1 among young students has increased rapidly. According to the National Center for AIDS/STD Control and Prevention, Chinese Center for Disease Control and Prevention (CDC), from 2008 to 2017 , the number of young students (aged 15-24) infected with HIV-1 increased by $63.8 \%$ annually, reaching 3,077 in $2017 .^{6}$ Sexual intercourse with male was the primary risk factor for HIV-1 infection among students. ${ }^{7}$ Several studies reported that the HIV-1 prevalence among men who have sex with men (MSM) attending university was over $5 \%$, and even up to $10 \%$ in some cities in China. ${ }^{8,9}$

\footnotetext{
${ }^{1}$ Key Laboratory of Environmental Medicine Engineering, Department of Epidemiology and Health Statistics, School of Public Health, Southeast University, Ministry of Education, Nanjing, Jiangsu, China.

${ }^{2}$ Institute for STI and HIV Control and Prevention, Nanjing Municipal Center for Disease Control and Prevention, Nanjing, Jiangsu, China.

${ }^{3}$ Department of Lung Transplant Center, The Affiliated Wuxi People's Hospital of Nanjing Medical University, Wuxi, China.

*These authors contributed equally to this work.
} 
Our previous study showed that CRF01_AE was the most prevalent strain in China, followed by CRF07_BC, subtype B'/B, CRF08_BC, subtype C, unique recombinant forms (URFs), and other circulating recombinant forms (CRFs). ${ }^{10}$ The subtype distribution of HIV-1 was different by areas, and HIV-1 genotypes possibly changed accordingly with the shift of transmission modes. Rapid changes in the HIV1 subtypes and seven independent clusters of HIV-1 strains were founded in young MSM in China, ${ }^{11}$ and notably, the complexity and diversity of HIV-1 strains appear to increase among young people. ${ }^{12}$ However, little is known about the molecular and genetic diversity of HIV-1 among college students in China. In this study, we conducted an HIV-1 molecular epidemiological investigation in Nanjing city, a gathering place of college students in China (with 75 institutions of higher education and nearly 780,000 students ${ }^{13}$ ), to characterize the genetic diversity of HIV-1 among college students.

\section{Materials and Methods}

\section{Ethics statement}

This study was reviewed and approved by the Human Research Ethics Committee of the Zhongda hospital affiliated Southeast University, China (approval ID: 2017ZDKYSB045). The objectives and the procedure of the study, and the potential risks and benefits of participating in the study were told to potential participants during the recruitment. Verbal or written informed consent were obtained from participants and they had the right to discontinue the survey at any time. All experiments were performed in accordance with the approved guidelines.

\section{Study subjects}

All newly diagnosed HIV-1-positive individuals were recruited consecutively between September 1, 2015, and June 30, 2019 in five districts (Gulou, Xuanwu, Jiangning, Qinhuai, and Qixia) of Nanjing, Jiangsu province, China. Students infected with HIV-1 in these five counties have accounted for $\sim 94 \%$ of all HIV-1-infected students in Nanjing in the past few years (unpublished data from Nanjing CDC). People were eligible if they met the following criteria: (1) aged 18 years and above; (2) self-reported as students and were confirmed by the student cards/identification card/residence permit; and (3) agreed to participate in this study with verbal or written informed consents. Eventually, 241 HIV-1positive students and 772 nonstudents were enrolled in the survey.

\section{Sample collection}

Peripheral blood samples $(10 \mathrm{~mL})$ were collected from each participant at the first follow-up after confirmation of HIV-1 infection. All participants were treatment-naïve when sampling and had no previous exposure to the highly active antiretroviral therapy. Sociodemographic data, including age, gender, marital status, education status, occupation, route of infection, and others were collected through a questionnaire survey. A molecular algorithm of a frequency of ambiguous calls in bulk sequencing of the pol gene under $0.5 \%$ was used to define a recent infection event $<1$ year before sampling. ${ }^{14}$

\section{HIV-1 RNA extraction and pol fragment amplification}

Plasma was separated from the whole blood within $12 \mathrm{~h}$ after sampling and stored at $-80^{\circ} \mathrm{C}$. HIV-1 RNA was extracted from $200 \mu \mathrm{L}$ of plasma specimens using the QIAamp Viral RNA Mini kit (Qiagen, Valencia, CA) according to the manufacturer's instructions, and was then applied in the subsequent reverse transcription-polymerase chain reaction (PCR) and nested PCR (nPCR) to generate the pol fragments. The pol fragments covered the entire protease and the first 300 codons of the reverse transcriptase (RT) gene. The reverse transcription PCR was performed using One Step RT-PCR kit with Super Script III Reverse Transcriptase (Invitrogen, Valencia, CA), and the nPCR was performed using the Ex Taq DNA Polymerase (Takara, Dalian, China). The primer pairs, condition, and the procedure of reverse transcription PCR and nPCR reactions were performed according to the Guidelines for HIV-1 Genotype Drug Resistance Detection and Quality Assurance (2013) issued by the National Center for AIDS/STD Control and Prevention, China CDC. ${ }^{15}$ The amplified products were sent to Wuhan Gene Create Biological Engineering Co., Ltd. for sequencing using ABI 3730XL automated DNA sequencers. Possible contaminations were excluded by using HIV-BLAST search (www.hiv.lanl .gov/content/sequence/BASCI_BLAST/basic_blast.html) and ElimDupes tool (https://www.hiv.lanl.gov/content/sequence/ elimdupesv2/elimdupes.html). All assembled sequences were submitted to the HIV-1 Sequence Quality Control Tool (https:// www.hiv.lanl.gov/content/sequence/QC/index.html) to confirm sequence quality.

\section{HIV-1 genotyping and phylogenetic analysis}

Rapid genotyping was performed using HIV-BLAST search. The reference sequences including subtypes A-D, F-H, J, K, CRF01_AE, CRF07_BC, CRF08_BC, CRF55_01B, CRF58_01B, CRF67_01B, and CRF68_01B were downloaded from Los Alamos HIV Databases (https://www.hiv .lanl.gov/content/sequence/NEWALIGN/align.html) based on the results of rapid genotyping. The obtained sequences were aligned together with reference sequences using the Clustal W program implemented in MEGA 7.0, and then manually edited. An approximately maximum likelihood phylogenetic tree was built using FastTree 2.3 software, with general time reversible model plus a gamma distribution among site rate heterogeneity $(\mathrm{GTR}+\mathrm{G}+\mathrm{I})$ nucleotide substitution model to determine HIV-1 genotypes.

\section{Recombination analysis}

A neighbor-joining ( $\mathrm{NJ}$ ) tree was constructed in MEGA 7.0 using the Kimura-two parameter model with 1,000 bootstrap replications to explore the phylogenetic characteristic of URFs. The potential inter-genotype recombination was first analyzed using the jumping profile Hidden Markov Model (jpHMM-HIV) tool (http://jphmm.gobics.de) and the Recombination Identification Program (RIP, https://www.hiv .lanl.gov/content/sequence/RIP/RIP.html), and was further confirmed with the SimPlot (version 3.5.1; http://sray.med .som.jhmi.edu/RaySoft/SimPlot/). The bootscan analysis was performed using a NJ tree with the Kimura-two parameter mode, 1,000 bootstrap replicates, $200 \mathrm{bp}$ window size, and 20 bp step. 


\section{Bayesian phylogeographic inference for URFs}

To explore the phylogenetic characteristics of the URFs among students in Nanjing, Bayesian phylogeographic inference was conducted using BEAST v.1.7.2 for URFs of Nanjing and reference sequences. The reference sequences were selected based on the highest similarities to all URFs in Nanjing through a BLAST search in HIV Sequence Database of the Los Alamos National Laboratory (LANL, https://www.hiv.lanl.gov/). ${ }^{16}$ Rates of evolution (in units of nucleotide substitutions/site/year) with 95\% Bayesian credible interval (BCI) and the time to the most recent common ancestor (tMRCA) were estimated simultaneously. States.set.prob analysis was used to infer the posterior probability (PP) support for the location estimates. ${ }^{17}$ The Bayesian inference was implemented under the GTR+I+ $\mathrm{\Gamma} 4$ nucleotide substitution model. Two molecular clock methods, including lognormal relaxed clock (Uncorrelated) and exponential relaxed clock (Uncorrelated), and two demographic modes, including constant size and Bayesian skyline model were selected and compared the heterogeneity. The Markov chain Monte Carlo analysis was computed for 100 million generations and sampled every 1,000 steps. The output was assessed for convergence by means of effective sampling size (ESS) after a 20\% burn-in using Tracer (http://tree.bio.ed.ac.uk/ software/tracer/). To minimize the effects of standard errors, only outputs with an ESS $>200$ were included. Maximum clade credibility trees were summarized using posterior distribution generated with TreeAnnotator and visualized in FigTree v1.4.3.

\section{Drug resistance analysis}

Viral pol gene sequences were submitted to the Stanford University HIV Drug Resistance Database (version 8.8, https://hivdb.stanford.edu/hivdb/by-sequences/) and the updated guidelines from the International AIDS Society Resistance Testing-USA panel was used to detect the primary drug resistance (DR) mutations. ${ }^{18}$ Transmitted DR mutations were analyzed using the calibrated population resistance tool according to the 2009 WHO surveillance drug resistance mutations list (http://cpr.stanford.edu/cpr.cgi). Levels of HIV-1 DR were evaluated by the Stanford DR algorithm.

Table 1. The Comparison of Sociodemographic Characteristics Between HIV-1-Positive Students and Nonstudents

\begin{tabular}{|c|c|c|c|c|c|}
\hline Variable & $\begin{array}{c}\text { Total }(\mathrm{N}=1,013), \\
\mathrm{n}(\%)\end{array}$ & $\begin{array}{c}\text { Students }(\mathrm{n}=241) \\
\mathrm{n}(\%)\end{array}$ & $\begin{array}{c}\text { Nonstudents }(\mathrm{n}=772) \\
\mathrm{n}(\%)\end{array}$ & $\chi^{2}$ value & $\mathrm{p}$ \\
\hline $\begin{array}{l}\text { Gender } \\
\text { Male } \\
\text { Female }\end{array}$ & $\begin{array}{r}958(94.57) \\
55(5.43)\end{array}$ & $\begin{array}{r}239(99.17) \\
2(0.83)\end{array}$ & $\begin{aligned} 719 & (93.13) \\
53 & (6.87)\end{aligned}$ & 13.030 & $<.001$ \\
\hline $\begin{array}{l}\text { Age }(\text { years, } \bar{x} \pm s) \\
<25 \\
25-34 \\
\geq 35 \\
\text { Unknown }\end{array}$ & $\begin{array}{c}325(32.08) \\
403(39.78) \\
272(26.86) \\
13(1.28)\end{array}$ & $\begin{aligned} 212 & (87.97) \\
29 & (12.03) \\
0 & (0.00) \\
0 & (0.00)\end{aligned}$ & $\begin{aligned} & 113(14.64) \\
& 374(48.45) \\
& 272(35.23) \\
& 13(1.68)\end{aligned}$ & 458.009 & $<.001$ \\
\hline $\begin{array}{l}\text { Infection routes } \\
\text { Sexual intercourse with male } \\
\text { Heterosexual behavior } \\
\text { Others } \\
\text { Unknown }\end{array}$ & $\begin{array}{l}752(74.23) \\
173(17.08) \\
23(2.27) \\
65(6.42)\end{array}$ & $\begin{aligned} & 216(89.63) \\
& 10(4.15) \\
& 2(0.83) \\
& 13(5.39)\end{aligned}$ & $\begin{array}{l}536(69.43) \\
163(21.11) \\
21(2.72) \\
52(6.74)\end{array}$ & 44.449 & $<.001$ \\
\hline $\begin{array}{l}\text { Registered residency } \\
\text { Nanjing } \\
\text { Other cities } \\
\text { Unknown }\end{array}$ & $\begin{array}{l}367(36.23) \\
548(54.10) \\
98(9.67)\end{array}$ & $\begin{aligned} 51(21.16) \\
174(72.20) \\
16(6.64)\end{aligned}$ & $\begin{array}{r}316(40.93) \\
374(48.45) \\
82(10.62)\end{array}$ & 41.984 & $<.001$ \\
\hline $\begin{array}{l}\text { Education (years) } \\
\quad<7 \\
7-9 \\
10-12 \\
\geq 13 \\
\text { Unknown }\end{array}$ & $\begin{array}{c}26(2.57) \\
107(10.56) \\
193(19.05) \\
6666(65.75) \\
21(2.07)\end{array}$ & $\begin{array}{c}0(0.00) \\
0(0.00) \\
0(0.00) \\
241(100.00) \\
0(0.00)\end{array}$ & $\begin{aligned} & 26(3.37) \\
& 107(13.86) \\
& 193(25.00) \\
& 425(55.05) \\
& 21(2.72)\end{aligned}$ & 164.765 & $<.001$ \\
\hline $\begin{array}{l}\text { Ethnicity } \\
\text { Han } \\
\text { Minority nationalities }\end{array}$ & $\begin{array}{r}974(96.15) \\
39(3.85)\end{array}$ & $\begin{array}{r}234(97.10) \\
7(2.90)\end{array}$ & $\begin{aligned} 740 & (95.85) \\
32 & (4.15)\end{aligned}$ & 0.764 & .448 \\
\hline $\begin{array}{l}\mathrm{CD}^{+}\left(\text {cells } / \mathrm{mm}^{3}\right) \\
\quad<200 \\
200-349 \\
350-499 \\
\geq 500 \\
\text { Unknown }\end{array}$ & $\begin{array}{l}163(16.09) \\
236(23.30) \\
246(24.28) \\
248(24.48) \\
120(11.85)\end{array}$ & $\begin{array}{l}20(8.30) \\
56(23.24) \\
72(29.88) \\
67(27.80) \\
26(10.78)\end{array}$ & $\begin{array}{r}143(18.52) \\
180(23.32) \\
174(22.54) \\
181(23.45) \\
94(12.17)\end{array}$ & 17.726 & .001 \\
\hline $\begin{array}{l}\text { Recent infection } \\
\text { Yes } \\
\text { No }\end{array}$ & $\begin{array}{l}647(68.47) \\
298(31.53)\end{array}$ & $\begin{array}{r}153(67.70) \\
73(32.30)\end{array}$ & $\begin{array}{l}494(68.71) \\
225(31.29)\end{array}$ & 0.081 & .776 \\
\hline
\end{tabular}

${ }^{\mathrm{a}} 945 \mathrm{pol}$ fragments were obtained from 1,013 plasma specimens. 


\section{Statistical analyses}

Statistical analyses were conducted using SPSS v20.0 (SPSS, Inc., Chicago, IL). Chi-square test was applied to compare the distribution of sociodemographic characteristics between students and nonstudents. Statistical significance was considered when $p$-value $<.05$ (two-tailed).

\section{Results}

\section{Sociodemographic characteristics}

Nearly $99 \%$ of students included in our study were male (239/241), with an average age of $21.70 \pm 2.65$ years (range 18$33)$. The vast majority of students $(216,89.63 \%)$ were infected through sexual intercourse with male. Only $21.16 \%$ (51) of students were native to Nanjing city, while $72.20 \%$ (174) were migrants from other cities. According to the molecular algorithm, $153(67.70 \%)$ students were classified as recent HIV-1 infection (Table 1). Compared to nonstudents, HIV-positive students were more likely to be male $(99.71 \%$ vs. $93.13 \%$; $\left.\chi^{2}=13.030, p<.001\right)$, infected via sexual intercourse with male $\left(89.63 \%\right.$ vs. $\left.69.43 \% ; \chi^{2}=44.449, p<.001\right)$, and reside in other cities instead of Nanjing (72.20\% vs. $48.45 \% ; \chi^{2}=41.984$, $p<.001)$.

\section{Prevalence of HIV-1 genotypes among students}

In total, $945 \mathrm{HIV}-1$ pol fragments covering $1,059 \mathrm{bp}$ (HXB2: 2,253-3,311), from 226 students and 719 nonstudents were obtained. There was no difference in the proportion of pol fragments successfully amplified between students $(93.77 \%, 226 / 241)$ and nonstudents $(93.12 \%, 719 /$ 772) $\left(\chi^{2}=0.121, p=.771\right)$. Based on the phylogenetic analysis (Fig. 1), one subtype, 6 CRFs, and several URFs were identified among 226 students in Nanjing. CRF01_AE was the most predominant genotype $(37.66 \%, 87 / 226)$, followed by CRF07_BC (32.90\%, 76/226), URFs (14.29\%, 33/ 226), CRF55_01B (5.63\%), CRF68_01B (3.46\%), CRF67_01B (3.03\%), subtype B (1.73\%), and CRF58_01B (1.30\%). The distribution of HIV-1 genotypes was similar between students and nonstudents (Supplementary Table S1). While subtype C, CRF08_BC, CRF59_01B, and CRF87_cpx were not found in students, a higher proportion of URFs was identified in students compared to nonstudents.

Combining the $\mathrm{NJ}$ tree analysis and recombination analysis for 33 pol fragments of URFs among students showed that these strains were descendent from subtype B, subtype C, CRF01_AE, and CRF07_BC. Among them, 16 (7.08\%) 01C_like strains displayed three different recombinant patterns, while 9 (3.98\%) 0107_like strains, 5 $(2.21 \%)$ 01BC_like strains and $3(1.33 \%)$ 01B_like strains shared the similar recombinant patterns, respectively (Fig. 2).

\section{Identification and phylogenetic characterization of URFs}

Due to the large number of URFs that appeared in students, a Bayesian phylogeographic inference was conducted to explore the phylogenetic characteristics of URFs among students. As shown in Table 2 and Supplementary Figure S1,
FIG. 1. Phylogenetic analysis of 1,007 pol sequences from Nanjing and references. The phylogenetic tree was constructed using the approximately maximum-likelihood method based on the pol region (HXB2: 2,253-3,311 nt) in Fast Tree 2.3. The nucleotide substitution mode was $\mathrm{GTR}+\mathrm{G}+\mathrm{I}$. The bootstrap value is indicated at relevant nodes. HIV-1 group $\mathrm{O}$ was chosen as an out-group in the rooted tree. The reference sequences including subtypes A-D, F-H, J, K, CRF01_AE, CRF07_BC, CRF55_01B, CRF58_01B， CRF67_01B, and CRF68_01B were from the Los Alamos HIV sequence database (http://hivweb.lanl.gov/content/index). The subtypes and circulating recombinant forms are marked in different shading.

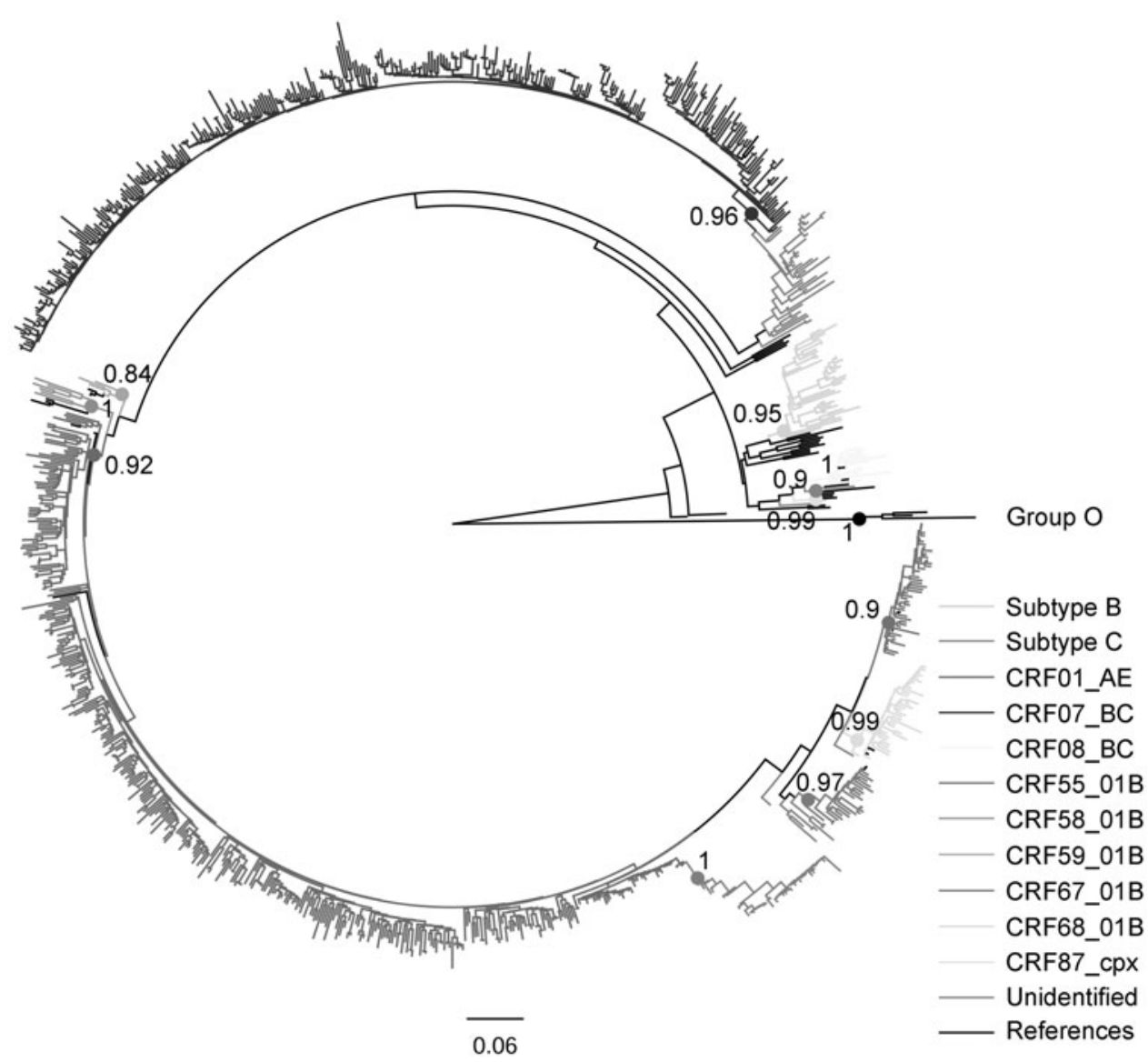




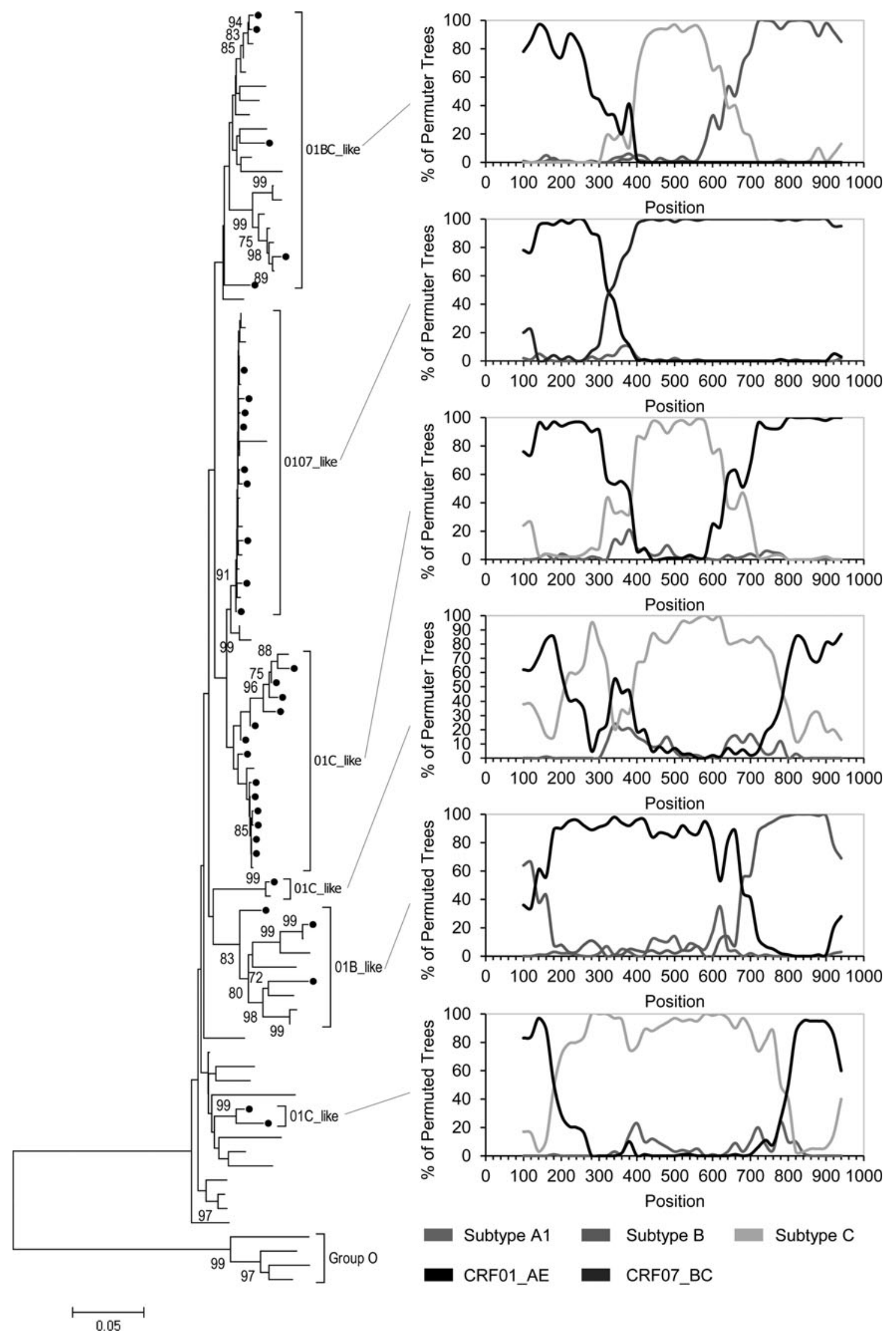

FIG. 2. Phylogenetic analysis and recombination analysis of $33 \mathrm{pol}$ sequences of unique recombinant forms in students. The phylogenetic tree was constructed in MEGA 7.0 using the Kimura-two parameter model with 1,000 bootstrap replications. The black nodes represent students. The recombination mode was analyzed using the SimPlot version 3.5.1. The bootscan analysis was performed using a neighbor-joining tree with the Kimura-two parameter mode, 1,000 bootstrap replicates, $200 \mathrm{bp}$ window size, and $20 \mathrm{bp}$ step. 


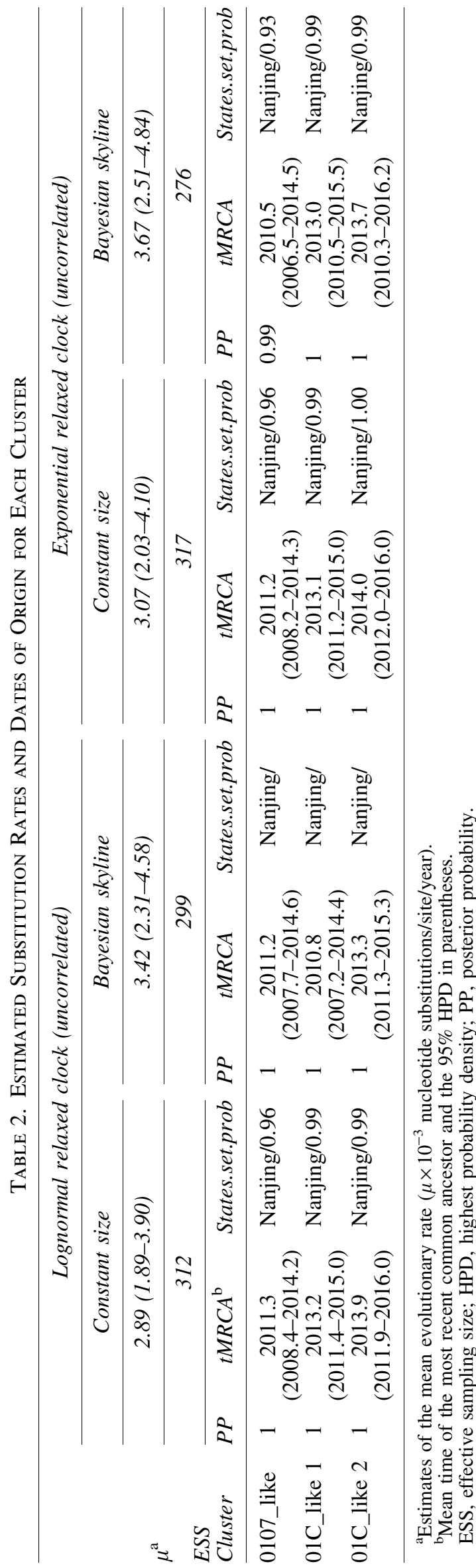

the estimated mean evolutionary rate was $2.89 \times 10^{-3}(95 \%$ BCI: 1.89-3.90) nucleotide substitutions/site/year. Approximately $64 \%$ (21/33) of URFs among students were located in three major clusters, and $36.4 \%$ (12/33) were scattered in several minimal clusters. Interestingly, the three major clusters were exclusively constructed of Nanjing sequences. The 0107_like cluster included 9 students and 15 nonstudents. The 01C_like 1 was comprised of 6 students and 2 nonstudents while 01C_like 2 clusters consisted of 6 students and 1 nonstudents. Of the strains in three major clusters, $87.5 \%$ (21/24), $87.5 \%(7 / 8)$, and $57.1 \%$ (4/7) were identified as new infection ( $<1$ year) in 0107_like, 01C_like 1, and 01C_like 2 cluster. The tMRCAs were 2011.3 (95\% BCI: 2008.4-2014.2), 2013.2 (95\% BCI: 2011.4-2015.0), and 2013.9 (95\% BCI: 2011.9-2016.0) for 0107_like, 01C_like 1, and 01C_like 2 cluster, respectively, and the mean genetic distances were $0.008 \pm 0.001,0.004 \pm 0.001$, and $0.017 \pm 0.002 \mathrm{nt}$ substitutions per site correspondingly. With the evidence from location estimates, we speculated that strains in these three clusters all originated from Nanjing ( $\mathrm{PP} \geq 0.99$, state probability $\geq 0.9$ ). The tMRCAs and state probability have no significant difference by clusters using various molecular clock methods and demographic modes.

\section{Drug-resistant mutations in students}

Among 226 students with pol segments, 36 (15.93\%) were identified to harbor HIV strains with at least one primary DR mutations. The prevalence of DR mutations to non-nucleoside reverse transcriptase inhibitors (NNRTIs) was $14.16 \%$ (32), followed by protease inhibitors (PIs) at $1.33 \%$ (3), and nucleoside reverse transcriptase inhibitors (NRTIs) at $0.88 \%$ (2). One $(0.44 \%)$ student was found to harbor dual-classes DR mutations. A total of 9 (3.98\%) students harbored transmitted DR mutations, including M46I (1), M46L (1), and L90LM (1) in PI area and L210W (1), K103N (1), K103S (2), and G190A (2) in RT area. There were no significant differences in the prevalence of primary and transmitted DR mutations in nonstudents and students $(p>.05)$ (Table 3$)$.

\section{Discussion}

The epidemic of HIV-1 among college students has drawn much attention by the Chinese government and society. Although several measures, including comprehensive sex education and more accessible HIV testing on campuses, have been implemented for controlling HIV-1 spread among students, the molecular characteristics of HIV-1 in this population were not well defined. Thus, this study investigated the HIV-1 genotypes and genetic diversity among college students in Nanjing. CRF01_AE and CRF07_BC were the two most dominant genotypes circulating among students in Nanjing, which was consistent with the studies conducted in Beijing and Kunming. ${ }^{19,20}$ Nonetheless, compared with one study in Hebei, ${ }^{21}$ our study indicated a lower proportion of subtype $B$ and a higher proportion of URFs among students in Nanjing. We also found that the proportion of CRF55_01B was higher in Nanjing students than those in Beijing and Hebei. Interestingly, CRF08_BC, which is a prevalent genotype $(11.1 \%)$ among heterosexuals in Jiangsu ${ }^{22}$ and nationwide, ${ }^{23}$ was not detected among students in Nanjing (the 
Table 3. The Comparison of Drug-Resistant Mutations Between HiV-1-Positive STUDENTS AND NONSTUDENTS

\begin{tabular}{|c|c|c|c|c|c|}
\hline Variable & $\begin{array}{c}\text { Total }(\mathrm{N}=945) \\
\mathrm{n}(\%)\end{array}$ & $\begin{array}{c}\text { Students }(\mathrm{n}=226), \\
\mathrm{n}(\%)\end{array}$ & $\begin{array}{l}\text { Nonstudents }(\mathrm{n}=719) \\
\mathrm{n}(\%)\end{array}$ & $\chi^{2}$ value & $\mathrm{p}$ \\
\hline Primary drug resistance & $132(13.97)$ & $36(15.93)$ & $96(13.35)$ & \multirow[t]{3}{*}{0.950} & \multirow[t]{3}{*}{.330} \\
\hline One-class & $125(13.23)$ & $35(15.49)$ & $90(12.52)$ & & \\
\hline Dual-classes & $7(0.74)$ & $1(0.44)$ & $6(0.83)$ & & \\
\hline PIs & $10(1.06)$ & $3(1.33)$ & $7(0.97)$ & \multirow[t]{3}{*}{ - } & \multirow[t]{3}{*}{$.710^{\mathrm{a}}$} \\
\hline M46I/L/MI/ML & $9(0.95)$ & $2(0.88)$ & $7(0.97)$ & & \\
\hline L90LM & $1(0.11)$ & $1(0.44)$ & & & \\
\hline NRTIs & $13(1.37)$ & $2(0.88)$ & $11(1.53)$ & \multirow[t]{12}{*}{ - } & \multirow[t]{12}{*}{$.774^{\mathrm{a}}$} \\
\hline A62 & $2(0.21)$ & & $2(0.28)$ & & \\
\hline D67DE/N & $4(0.42)$ & & $4(0.56)$ & & \\
\hline $\mathrm{K} 65 \mathrm{KR} / \mathrm{R}$ & $2(0.21)$ & & $2(0.28)$ & & \\
\hline $\mathrm{K} 70 \mathrm{~T} / \mathrm{R}$ & $2(0.21)$ & $1(0.44)$ & $1(0.14)$ & & \\
\hline K219E & $1(0.11)$ & & $1(0.14)$ & & \\
\hline L74I & $1(0.11)$ & & $1(0.14)$ & & \\
\hline L210 LW/W & $2(0.21)$ & $1(0.44)$ & $1(0.14)$ & & \\
\hline M184V & $2(0.21)$ & & $2(0.28)$ & & \\
\hline $\mathrm{T} 215 \mathrm{~F}$ & $1(0.11)$ & & $1(0.14)$ & & \\
\hline V75I & $1(0.11)$ & & $1(0.14)$ & & \\
\hline Y115YF & $1(0.11)$ & & $1(0.14)$ & & \\
\hline NNRTIs & $116(12.27)$ & $32(14.16)$ & $84(11.68)$ & \multirow{12}{*}{0.979} & \multirow[t]{12}{*}{.332} \\
\hline E138A/EA/EG/G & $9(0.95)$ & $3(1.33)$ & $6(0.83)$ & & \\
\hline F227FL/L & $2(0.21)$ & & $2(0.28)$ & & \\
\hline G190GE/A & $5(0.53)$ & $3(1.33)$ & $2(0.28)$ & & \\
\hline K101E/H & $2(0.21)$ & & $2(0.28)$ & & \\
\hline $\mathrm{K} 103 \mathrm{KQ} / \mathrm{N} / \mathrm{Q} / \mathrm{S}$ & $11(1.16)$ & $4(1.77)$ & $7(0.97)$ & & \\
\hline L100LI & $1(0.11)$ & & $1(0.14)$ & & \\
\hline M230MI & $1(0.11)$ & & $1(0.14)$ & & \\
\hline V106I/M/VEKM/VI & $20(2.12)$ & $4(1.77)$ & $16(2.22)$ & & \\
\hline V108I & $2(0.21)$ & $1(0.44)$ & $1(0.14)$ & & \\
\hline V179D/E/T/VD & $75(7.94)$ & $23(10.18)$ & $52(7.23)$ & & \\
\hline Y181C & $2(0.21)$ & & $2(0.28)$ & & \\
\hline Transmitted drug resistance & $38(4.02)$ & $9(3.98)$ & $29(4.03)$ & 0.001 & .973 \\
\hline
\end{tabular}

${ }^{a}$ Fisher' s exact probability test.

NRTI, nucleoside reverse transcriptase inhibitor; PI, protease inhibitor.

capital of Jiangsu Province). These differences in prevalence of specific genotypes between our study population and other populations may be because a large proportion of students recruited identified as MSM. Interestingly, the distribution of HIV-1 genotypes among students in Nanjing was similar to that among native nonstudents, suggesting the close transmission links between the two groups. Strengthening the implementation of HIV control measures among native nonstudents have the potential to curb the HIV-1 transmission in students as well.

Usually, URFs were identified in dually or multiply infected individuals among MSM. ${ }^{24,25}$ Unprotected anal sex with multiple partners may favor the generation of URFs. ${ }^{26,27}$ The high proportion of URFs and the diverse recombinant forms among students in Nanjing suggested that students tended to have high risk sexual behaviors. Furthermore, we speculated that some strains were transmitted into this population from nonstudents based on the results of Bayesian phylogeographic inference. As a coastal metropolis and an important transportation hub, Nanjing has attracted an increasing number of domestic migrant people in recent years. In this study, we found that more than three quarters of HIVinfected students and half of nonstudents were non-native that is, from other cities or provinces. These migrants might serve as a bridge that facilitates viral transmission from other cities/provinces to Nanjing. This could partly explain why such high prevalence of URFs and some rarely CRFs, for instance, CRF55_01B, CRF58_01B, CRF67_01B, and CRF68_01B, were identified in Nanjing. Notably, three large URFs clusters appeared and spread in both students and nonstudents in Nanjing. The low mean genetic distance and the high proportion of newly infected individuals in these clusters suggested that these strains might continue to expand among students in the future if intervention not being implemented.

The prevalence of transmitted DR mutations among students in Nanjing had approached the alert level (5.0\%) issued by $\mathrm{WHO},{ }^{28}$ which hinted that the continuous monitoring for transmitted DR mutations in students is necessary. The M46 was rarely reported in China, ${ }^{29}$ as PIs were not included in the first-line free ART and rarely used in China. However, two M46 mutations observed among students in Nanjing was suggestive of possible sexual promiscuity among the MSM students in Nanjing with individuals from other regions or countries where PIs are used widely.

This study has several limitations. First, we recruited study participants from five districts (12 districts in Nanjing in total), 
as they accounted for more than $90 \%$ of all HIV-1-infected students. However, nonstudents in our study might not be representative of its source population. Therefore, results of differences in HIV-1 characteristics between students and nonstudents might have been biased. Second, misclassification of students and nonstudents might exist as some HIV-positive college students concealed attending colleges for the consideration of privacy. Third, the identification of genotypes was based on pol fragments, instead of near full-length genomic (NFLG) sequences, resulting in an underestimation of the proportion of URFs. The recombinant forms for these URFs should be investigated further using NFLG sequences.

\section{Conclusions}

Our study described the molecular epidemiological features of HIV-1 among college students in Nanjing, China, and presented novel biological data for this population. These results provided critical information for designing effective interventions to limit HIV transmission among college students in Nanjing. The similar characteristics between students and nonstudents highlighted the importance of placing effective and efficient measures in nonstudents for controlling HIV-1 in students. Urgent implementation of comprehensive control measures is necessary to limit the severe epidemic of URFs in Nanjing.

\section{Authors' Contributions}

W.L., X.L., and P.W. conceived and designed the study. Y.G., Y.X., S.W., Y.H., Q.N., Y.L., and R.L. did the data entry and management of the database. W.L., Z.Z., P.W., and X.L. analyzed the data. W.L., J.C., and X.L., wrote the article of the report with input from other authors. All authors read and approved the final article.

\section{Acknowledgments}

The authors gratefully acknowledgement the staff in Jiangsu CDC, and Nanjing CDC for patient recruitment and blood samples collection.

\section{Author Disclosure Statement}

No competing financial interests exist.

\section{Funding Information}

This study was funded by the Humanities and Social Sciences of Ministry of Education Planning Fund of China (no. 16YJA840014). The funders had no role in the study design, data collection and analysis, decision to publish, or preparation of the article.

\section{Supplementary Material}

Supplementary Figure S1

Supplementary Table S1

\section{References}

1. AVERT: Young people, HIV and AIDS. Available at https://www.avert.org/professionals/hiv-social-issues/keyaffected-populations/young-people accessed December 1, 2019.
2. UNICEF: Global and regional trends. Available at https:// data.unicef.org/topic/hivaids/global-regional-trends/ accessed December 1, 2019.

3. UNICEF: Adolescent deaths from AIDS tripled since 2000 UNICEF. Available at https://www.unicef.org/media/media_ 86384.html accessed December 1, 2019.

4. UNICEF: The state of the world's children 2017 statistical tables. Available at https://data.unicef.org/resources/stateworlds-children-2017-statistical-tables/ accessed December $1,2019$.

5. Xu J-J, Reilly KH, Lu C-M, et al.: A cross-sectional study of HIV and syphilis infections among male students who have sex with men (MSM) in northeast China: Implications for implementing HIV screening and intervention programs. BMC Public Health 2011;11:287.

6. National Center for AIDS/STD Control and Prevention China CDC: A regular news conference of China's national health commission on nov 23, 2018. Available at http://ncaids .chinacdc.cn/zxzx/mtsd3/201811/t20181123_197487.htm accessed December 5, 2019.

7. Zou H, Tucker JD, Fan S, et al.: Learning about HIV the hard way: HIV among Chinese MSM attending university. Lancet Infect Dis 2018;18:16-18.

8. Li Y, Xu J, Reilly KH, et al.: Prevalence of HIV and syphilis infection among high school and college student MSM in China: A systematic review and meta-analysis. PLoS One 2013;8:e69137.

9. Xu Y, Zhu Z, Wu S, Liu L, Guo L, Zhang M: Analysis on the characteristics of AIDS epidemic among young students in nanjing from 2002 to 2014 (in Chinese). Chin J AIDS STD 2015:893-894.

10. Li X, Li W, Zhong P, et al.: Nationwide trends in molecular epidemiology of HIV-1 in China. AIDS Res Hum Retroviruses 2016;32:851-859.

11. Li Z, Liao L, Feng Y, et al:: Trends of HIV subtypes and phylogenetic dynamics among young men who have sex with men in China, 2009-2014. Sci Rep 2015;5:16708.

12. Zhang W, Chen J, Pan X, et al.: Trends of HIV-1 subtypes among young people in Hangzhou, China. AIDS Res Hum Retroviruses 2017;33:219-227.

13. NJEDU (2018): Digital report of Nanjing education development in 2017. Available at http://edu.nanjing.gov .cn/zwgk/tjsjjjd/201904/t20190412_1506528.html accessed December 20, 2019.

14. Kouyos RD, von Wyl V, Yerly S, et al:: Ambiguous nucleotide calls from population-based sequencing of HIV-1 are a marker for viral diversity and the age of infection. Clin Infect Dis 2011;52:532-539.

15. China CDC: Guideline for HIV-1genotyping drug resistance testing and quality assurance. Available at www.cqcdc.org/ $\mathrm{html} /$ content/18/01/2656.shtml accessed December 20, 2019.

16. Takebe $\mathrm{Y}$, Naito $\mathrm{Y}$, Raghwani J, et al.: Intercontinental dispersal of HIV-1 subtype B associated with transmission among men who have sex with men in Japan. J Virol 2014; 88:9864-9876.

17. Faria NR, Rambaut A, Suchard MA, et al.: HIV epidemiology. The early spread and epidemic ignition of HIV-1 in human populations. Science 2014;346:56-61.

18. Wensing AM, Calvez V, Gunthard HF, et al.: 2017 Update of the drug resistance mutations in HIV-1. Top Antivir Med 2016;24:132-133.

19. Hao M, Wang J, Xin R, et al.: Low rates of transmitted drug resistances among treatment-naive HIV-1-infected students in Beijing, China. AIDS Res Hum Retroviruses 2017;33:970-976. 
20. Chen M, Ma Y, Su Y, et al.: HIV-1 genetic characteristics and transmitted drug resistance among men who have sex with men in Kunming, China. PLoS One 2014;9:e87033.

21. Lu X, Zhao C, Wang W, et al.: HIV-1 genetic diversity and its distribution characteristics among newly diagnosed HIV-1 individuals in Hebei province, China. AIDS Res Ther 2016; 13:3.

22. Xiao P, Zhou Y, Lu J, et al: HIV-1 genotype diversity and distribution characteristics among heterosexually transmitted population in Jiangsu province, China. Virol J 2019;16:51.

23. He X, Xing H, Ruan Y, et al.: A comprehensive mapping of HIV-1 genotypes in various risk groups and regions across China based on a nationwide molecular epidemiologic survey. PLoS One 2012;7:e47289.

24. Liu Y, Su B, Zhang Y, et al.: Brief report: Onward transmission of multiple HIV-1 unique recombinant forms among men who have sex with men in Beijing, China. J Acquir Immune Defic Syndr 2019;81:1-4.

25. Chen M, Ma Y, Chen H, et al:: Spatial clusters of HIV-1 genotypes in a recently infected population in Yunnan, China. BMC Infect Dis 2019;19:669.

26. Raifman J, Beyrer C, Arrington-Sanders R: HIV education and sexual risk behaviors among young men who have sex with men. LGBT Health 2018;5:131-138.
27. Peltzer K, Pengpid S: Risk and protective factors affecting sexual risk behavior among school-aged adolescents in Fiji, Kiribati, Samoa, and Vanuatu. Asia Pac J Public Health 2016;28:404-415.

28. Bennett DE, Camacho RJ, Otelea D, et al:: Drug resistance mutations for surveillance of transmitted HIV-1 drug-resistance: 2009 update. PLoS One 2009;4:e4724.

29. Li X, Xue Y, Lin Y, et al.: Evolutionary dynamics and complicated genetic transmission network patterns of HIV1 CRF01_AE among MSM in Shanghai, China. Sci Rep 2016;6:34729.

Address correspondence to: Pingmin Wei Department of Epidemiology and Health Statistics School of Public Health Southeast University 87 Dingjiaqiao Road Nanjing 210009

China

E-mail: mpw1963@126.com 